\author{
Vertical structure of pulsations in roAp stars \\ M. Sachkov, ${ }^{1}$ T. Ryabchikova, ${ }^{1,2}$ O. Kochukhov, ${ }^{3}$ D. Lyashko ${ }^{4}$ \\ ${ }^{1}$ Institute of Astronomy, Russian Academy of Science, 48 Pyatnitskaya str., 119017 Moscow, Russia \\ ${ }^{2}$ Institute of Astronomy, University of Vienna, 1180 Vienna, Austria \\ ${ }^{3}$ Department of Astronomy and Space Physics, Uppsala University, Box 515, SE-751 20 Uppsala, Sweden \\ 4 Tavrian National University, Yaltinskaya 4, 330000 Simferopol, Ukraine
}

\begin{abstract}
We present a detailed analysis of the vertical cross-section of the pulsation modes of roAp stars. We use unique properties of roAp stars, in particular their chemical stratification, to resolve the vertical structure of $p$-modes and to study the propagation of pulsation waves. The aim of this analysis is to derive a complete picture of the depth-dependence of amplitudes and phases of magnetoacoustic waves from the variability of hundreds of spectral lines of different elements/ions.
\end{abstract}

\title{
Spectroscopic observations
}

The main observational data set analysed in our study consists of 958 observations of eight roAp stars (HD 9289, HD 12932, HD 19918, HD 101065, HD 122970, HD 128898, HD 134214, HD 137949), obtained with the UVES instrument at the ESO VLT between October 8, 2003 and March 12, 2004 in the context of the observing program 072.D-0138. The ESO Archive facility was used to search and retrieve science exposures and the respective calibration frames. Observations of each target cover 2 hours and consist of an uninterrupted high-resolution spectroscopic time-series with a total number of exposures ranging from 69 to 265 . The length of individual exposures was $40^{\mathrm{s}}$ or $80^{\mathrm{s}}$, except for the brightest roAp star HD 128898 ( $\alpha$ Cir), for which a $1.5^{\mathrm{s}}$ exposure time was used. Details of these observations are given by Kurtz et al. (2006).

In addition, we used the observations of HD 24712 obtained on November 11, 2004 in the DDT program 274.D-5011 (92 time-resolved spectra collected with UVES) and 70 observations of HD 201601 ( $\gamma$ Equ) obtained on 19 August, 2003 with the NES spectrograph attached to the 6-m telescope of the Russian Special Astrophysical Observatory.

\section{Phase-amplitude diagrams}

The radial velocities were measured with a centre-of-gravity technique. We used only unblended or minimally blended lines. It was shown (Sachkov et al. 2006) that a model of nonadiabatic nonradial pulsations (Saio 2005) calculated for the roAp star HD 24712 roughly explains amplitude and phase changes from the photospheric level up to $\log \tau_{5000}=-4$ : amplitude and phase increase towards the outer layers. Model calculations do not extend above this level, but observations show that the phases continue to increase gradually from one element/ion to another (see also Fig. 1 by Kochukhov 2007). This was interpreted as a propagation of the pulsation wave through the stellar atmosphere: the later in time a pulsation wave reaches its maximum, the higher in the atmosphere a chemical element is concentrated. Consequently, the pulsation phase can be used to study the vertical structure of pulsation modes. 
We propose to use the phase-amplitude diagrams as a first step in the interpretation of roAp pulsational observations. Such an approach has an advantage of being suitable to compare the pulsational behaviour of different elements, while the phase/amplitude dependence on the line intensity may be applied to one element only because of the chemical stratification. This analysis requires accurate measurements of both amplitudes and phases of many lines including weak ones. Therefore, our sample was limited to slowly rotating roAp stars.

In all stars we have detected pulsational variability in the lines of the rare-earth elements (REE), which show maximum radial velocity (RV) amplitude. Except 33 Lib, no pulsations were detected in the lines of the elements lighter than Sr. The lowest significant RV amplitudes were derived for the YII lines. Finally, for the first time we found pulsations in doublyionized thorium lines in four coolest roAp stars of our sample: HD 101065, HD 122970, HD 24712 and HD 134214. Similar to REEs, thorium shows a characteristic abundance anomaly: a 1-2 dex difference in the element abundance derived from the lines of the first and second ions. We attribute this anomaly to a strong vertical stratification similar to REEs a layer with $4-5$ dex overabundance above $\log \tau_{5000}=-4$. At present thorium is the heaviest element with this kind of stratification which shows measurable pulsation amplitudes.

Although the overall pulsational behaviour of roAp stars is different, we found certain common features. The phase shifts of the RV curves are arranged in the following sequence:

- The lowest RV amplitudes are detected in the layers of the EuII (and Fe in $33 \mathrm{Lib}$ ) line formation, then they go through the layers where the $\mathrm{H \alpha}$ core, $\mathrm{Nd}$ and $\mathrm{Pr}$ lines are formed, reach maximum and after that, show a decrease of amplitude in most stars;

- the phases of the RV curves of the first ions are always followed by the second ones;

- the largest phase shifts are detected in TbIII and ThiII lines;

- in the atmospheres of roAp stars with pulsation frequencies much below the cut-off frequency, the pulsations have a standing wave character in the deeper layers and then behave like a running wave in the outer layers. In three stars: HD 24712, HD 134214, $\alpha$ Cir, which have pulsation frequency close to (or even higher than) the cut-off frequency, the pulsation wave is running from the deeper layers.

- the YII lines show the lowest detectable RV amplitudes. However, their phases differ by $\approx 0.5$ periods from other weakly pulsating lines. This may be an indication of the existence of pulsation node in deep photospheric layers, in agreement with theoretical predictions.

Acknowledgments. This work was supported by the RFBR grant 04-02-16788, by the Presidium RAS program 'Non-stationary phenomena in astronomy'. MS also gratefully acknowledges the support of RF president grant MK-954.2005.2.

\section{References}

Kochukhov O., 2007, Comm. Asteroseis., 150, 39

Kurtz D. W., Elkin V. G., Mathys G., 2006, MNRAS, 370, 1274

Sachkov M., Ryabchikova T., Bagnulo S., et al., 2006, Mem. Soc. Astron. Ital., 77, 397

Saio H., 2005, MNRAS, 360, 1022 